\title{
The artificial somato-autonomic reflex arch does not improve bowel function in subjects with spinal cord injury
}

\author{
MM Rasmussen ${ }^{1,2}, \mathrm{~K} \mathrm{Krogh}^{3}$, D Clemmensen ${ }^{2}, \mathrm{H}_{\text {Tankisi }}^{4}$, A Fuglsang-Frederiksen ${ }^{4}, \mathrm{Y}$ Rawashdeh $^{5}$, \\ $\mathrm{H}_{\text {Bluhme }}{ }^{6}$ and P Christensen ${ }^{1}$
}

Study design: Prospective cohort study.

Objective: Although introduced for neurogenic bladder dysfunction, it has been suggested that the artificial somato-autonomic reflex arch alleviates neurogenic bowel dysfunction (NBD). We aimed at evaluating the effects of the reflex arch on NBD.

Setting: Denmark.

Methods: Ten subjects with supraconal spinal cord injury (SCI) (nine males, median age 46 years) had an anastomosis created between the ventral part of the fifth lumbar or first sacral nerve root and the ventral part of the second sacral nerve root. Standardized assessment of segmental colorectal transit times with radiopaque markers, evaluation of scintigraphic assessed colorectal emptying upon defecation, scintigraphic assessment of colorectal transport during stimulation of the reflex arch, standard anorectal physiology tests and colorectal symptoms were performed at baseline and 18 months after surgery.

Results: No significant change was observed in colorectal emptying upon defecation (median $31 \%$ of the rectosigmoid at baseline vs $75 \%$ at follow-up, $P=0.50$ ), no movement of colorectal contents was observed during stimulation of the reflex arch. Segmental colorectal transit times, anal sphincter pressures and rectal capacity did not change, and no change was seen in NBD score (median 13.5 (baseline) vs 12.5 (follow-up), $P=0.51$ ), St Marks fecal incontinence score (4.5 vs 5.0, $P=0.36$ ) and Cleveland constipation score (6.0 vs $8.0, P=0.75)$.

Conclusions: The artificial somato-autonomic reflex arch has no effect on bowel function in subjects with supraconal SCl.

Spinal Cord (2015) 53, 705-710; doi:10.1038/sc.2015.75; published online 28 April 2015

\section{INTRODUCTION}

Spinal cord injury (SCI) has profound impact on bowel function leading to neurogenic bowel dysfunction (NBD) in the majority of subjects resulting in severe constipation, abdominal pain and fecal incontinence. ${ }^{1}$ Consequently, NBD ranks among the three complications most severely affecting the quality of life of subjects surviving SCI. Conservative management is preferred when possible, however, surgical procedures such as an appendicostomy for antegrade colonic irrigation or a colostomy may be indicated. In spite of recent advances in the treatment of NBD, a significant proportion of subjects with SCI continue to have severe symptoms.

The surgically created artificial somato-autonomic reflex arch is a novel and fundamentally different approach. It is reported to create a reflex arch from the dermatome of L5, through the spinal cord and further to the bladder and the distal colorectum. Following the procedure, frequent mechanical stimulation of the skin on the leg, typically by scratching, is supposed to initiate voiding and may simultaneously promote propulsion of colorectal content. Initial reports have been encouraging with respect to improved bladder function. ${ }^{2}$ Recently, we have questioned the Chinese reports as we found no effect of the procedure with regards to neurogenic bladder dysfunction. ${ }^{3}$ It is also reported that bowel function improves from the procedure, but this has not been studied in detail. ${ }^{2,4,5}$ This calls for a detailed study of the effects of the Xiao procedure on NBD.

The aim of the present study was to provide a comprehensive state-of-the-art evaluation of the effects of the somato-autonomic reflex arch on bowel function in a small group of well-characterized subjects with supraconal SCI. We hypothesized that frequent stimulation of the artificial somato-autonomic reflex arch would increase colorectal transport both during and between defecations thereby reducing colorectal transit time and improving bowel function.

\section{SUBJECTS AND METHODS}

Before initiating the present study, a multidisciplinary team from our unit went to China to see how the surgical procedure was performed.

All subjects included underwent thorough evaluation of colorectal, bladder and electrophysiological function at baseline and 18 months after surgery. Data on bladder are presented elsewhere. ${ }^{3}$ The local ethical committee gave project approval (number 20090113) and the project was registered on ClinicalTrials. gov (NCT01241630 and NCT01274312).

Our subject inclusion criteria were patients $\geqslant 18$ years of age, American Spinal Injury Association Impairment Scale score A or B, injury between spinal cord cervical level 4 and lumbar level 4, preserved medial hamstring reflex arch and suitable preoperative electrophysiological response from the relevant nerves

\footnotetext{
${ }^{1}$ Pelvic floor unit, Department of Surgery, Aarhus University Hospital, Aarhus, Denmark; ${ }^{2}$ Department of Neurosurgery, Aarhus University Hospital, Aarhus, Denmark; ${ }^{3}$ Neurogastroenterology Unit, Department of Hepatology and Gastroenterology, Aarhus University Hospital, Aarhus, Denmark; ${ }^{4}$ Department of Clinical Neurophysiology, Aarhus University Hospital, Aarhus, Denmark; ${ }^{5}$ Department of Urology, Aarhus University Hospital, Aarhus, Denmark and ${ }^{6}$ Department of Nuclear Medicine and PET Center, Aarhus University Hospital, Aarhus, Denmark

Correspondence: Dr MM Rasmussen, Department of Neurosurgery, Aarhus University Hospital, Noerrebrogade 44, building number 10, Aarhus C 8000, Denmark.

E-mail: mikkrs@rm.dk
}

Received 4 January 2015; revised 28 March 2015; accepted 1 April 2015; published online 28 April 2015 
ipsilateral to intended surgery. Exclusion criteria were respirator-dependent subjects, malignant urological disease, pharmaceutical treatment affecting bowel dysfunction that could not be paused for at least $24 \mathrm{~h}$ before the scintigraphic investigations, ileostomy, colostomy and pregnancy or planned pregnancy. ${ }^{3}$

The surgical procedure was carried out in accordance with in-theatre experiences attained in China and as described by Xiao et al..$^{2}$ Surgery was performed by an experienced neurosurgeon with firsthand experience from the China visit. Pre- and perioperatively, electrophysiological evaluation helped determine surgical anastomotic level and laterality. Subjects underwent a hemilaminectomy of L4 and L5 and decompression of the sacral bone. Under microscope magnification, the dura was opened and the fifth lumbar (L5) and first (S1) and second (S2) sacral roots were identified ipsilaterally using anatomical localization and intraoperative electrophysiology. S2 was chosen for anastomosis as satisfactory electrophysiological response was obtained in all cases. The ventral (motor) root filaments were separated from the dorsal (sensory) and tested with electrophysiology. The dorsal filaments were kept intact, while the ventral roots (or part of one) were transsected and a microanastomosis was created between the L5 (in one case S1) and S2 by suturing the proximal lumbar ventral L5 and distal ventral S2 nerve root sheath aligned to dura (Figure 1). Owing to dural opening, 3 days post-surgery, flat bed rest was instituted. Afterwards, mobilization started slowly and when mobilized, the patients were discharged. Prior to surgery and investigations, Baclofen was paused for $24 \mathrm{~h}$ (Figure 1). ${ }^{3}$

To rule out polyneuropathy, entrapment neuropathy and other kind of peripheral nerve lesion, to ensure correct nerve filament selection during the surgical procedure and to assess reinnervation at follow-up, electrophysiological evaluations were performed at baseline, during the surgical procedure and at follow-up. Two experienced neurophysiology consultants performed all electrophysiological tests using Keypoint equipment (Medtronic, Copenhagen, Denmark). ${ }^{3}$

On the basis of previous reports, reinnervation was expected to have occurred and the artificial somato-autonomic reflex arch expected to be functional approximately 12 months after surgery. ${ }^{2}$

As proposed by the inventor of the procedure, frequent stimulation by scratching the appropriate sensory dermatome was encouraged from 8 months post surgery and onwards ${ }^{2}$ in 30 -s bouts ideally prior to bladder emptyings daily or some attempts each week.

\section{Total gastrointestinal and segmental colonic transit times}

We used the method described by Abrahamsson et al. ${ }^{6}$ Thus, subjects took 10 radiopaque markers each day on six consecutive days. On day 7, an abdominal $\mathrm{X}$-ray was taken and total gastrointestinal time as well as segmental colonic transit times were calculated from the markers present.

\section{Scintigraphic evaluation of colorectal transport}

Our protocol for scintigraphic evaluation of colorectal emptying during defecation has previously been described in detail. ${ }^{7,8}$ To mark colorectal contents within the whole colorectum, subjects swallowed two doses of

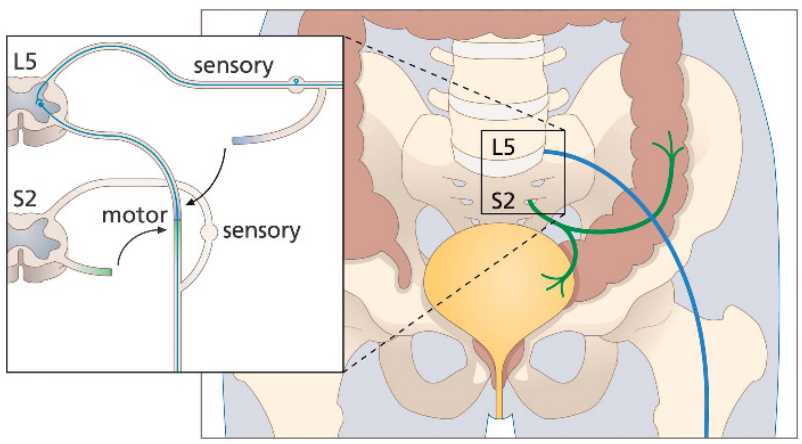

Figure 1 The somato-autonomic reflex arch is formed through an intradural surgical procedure creating an anastomosis between the ventral (motor) part of fifth lumbar nerve root and the ventral part of the first sacral nerve root sheath. Reprinted with permission. ${ }^{3}$
${ }^{111}$ In-coated resin pellets adding up to a maximum total dose of $4 \mathrm{MBq}$. If gastrointestinal time was less than 2.5 days, pellets were taken 48 and $24 \mathrm{~h}$ before scintigraphy. If gastrointestinal time was more than 2.5 days, the pellets were taken 72 and $24 \mathrm{~h}$ before scintigraphy. Baclofen was paused $24 \mathrm{~h}$ before study. Subjects arrived at our department of nuclear medicine in the morning after an overnight fast and without customary morning defecation. Initially a baseline scintigram was aquired (acquisition time was $10 \mathrm{~min}$, five 2-min frames). Afterwards, the artificial somato-autonomic reflex arch was stimulated for $10 \mathrm{~min}$ by scratching the dermatome. During stimulation, continuous 10-s scintgraphic frames were obtained to record any movement of stools within the colorectum. Following this, subjects consumed a standardized breakfast to activate the gastro-colic response. Finally, after subjects having performed their normal bowel emptying procedure, the last scintigram was recorded (10 $\mathrm{min}$, five 2-min frames).

Four colorectal segments were defined: (i) the cecum and ascending colon including the right flexure, (ii) the transverse colon, (iii) the descending colon including the left flexure and (iv) the recto-sigmoid. The geometric mean was calculated correcting for tissue attenuation by computing the square root of each segments anterior and posterior counts. ${ }^{9}$ Colorectal emptying and the movement of content during defecation were assessed by means of the previously published defecation score by comparing the scintigrams before and after defecation. ${ }^{10}$ For example, a defecation score of 40 corresponds to emptying $40 \%$ of the recto-sigmoid, while 150 equals emptying of the whole recto-sigmoid and $50 \%$ of descending colon. The maximum being 400 indicating that all colorectal content was emptied upon defecation. Antegrade and retrograde transport was calculated for each segment and reported in percentage of specific segment count prior to defecation.

The frames recorded during attempted reflex stimulation were used for dynamic assessment of colorectal transport as a direct result of stimulation. To ensure enough counts for analysis, the frames were merged to 30-s frames and analyzed using counts in the four predefined colorectal segments over time.

Data were discarded if colorectal segments overlapped on the scintigraphic frames as this makes delineation of segments impossible. Furthermore, to have sufficient counts for valid computation, segments included for analysis should have at least $7.5 \%$ of the total counts prior to defecation. The total effective whole body radiation dose ranged between 2.2 and $6.2 \mathrm{mSv}$ depending on the individual gastrointestinal time.

\section{Anorectal physiology tests}

We determined anal resting pressure as well as anal pressure during attempted squeeze. Maximum rectal capacity was defined as the volume where reflex expulsion of the rectal balloon occurred. Our protocol and normative data for anorectal physiology tests have previously been published. ${ }^{11}$

\section{Assessment of bowel symptoms}

The international bowel function basic and extended SCI data set with a few additional questions were used for description of bowel symptoms. ${ }^{12,13}$ On the basis of these, the NBD score, ${ }^{14}$ the St Marks incontinence score ${ }^{15}$ and the Cleveland constipation score ${ }^{16}$ were calculated for each subject.

\section{Statistical analysis}

Data were analyzed using the Wilcoxon signed rank test. Median and 25 and $75 \%$ quartiles are reported. $P<0.05$ was considered as statistically significant.

\section{RESULTS}

\section{Subjects}

Ten SCI subjects underwent the surgical procedure. A detailed description of the inclusion process is given elsewhere. ${ }^{3}$ Table 1 shows baseline data for individual subjects and Figure 2 give a schematic overview of subject inclusion.

\section{Surgical procedure and complications}

Eight subjects had the standard decided anastomotic procedure from left L5-S2. On the basis of electrophysiology tests, one subject had a right L5-S2 and one a right S1-S2 anastomosis instead. Median 
Table 1 Baseline subject data $(n=10)$

\begin{tabular}{|c|c|c|c|c|c|c|c|}
\hline Subject & $\begin{array}{l}\text { Age at surgery } \\
\text { (years) }\end{array}$ & Injury level & AIS score & $\begin{array}{c}\text { Time (injury—surgery) } \\
\text { (years) }\end{array}$ & $\begin{array}{l}\text { Time tofollow-up } \\
\text { (months) }\end{array}$ & $\begin{array}{c}\text { Bowel dysfunction unrelated } \\
\text { to } \mathrm{SCl}\end{array}$ & $\begin{array}{c}\text { Gastrointestinal surgical } \\
\text { procedures }\end{array}$ \\
\hline 1 & 19 & Th 10 & $A$ & 1 & 20 & No & No \\
\hline 2 & 22 & C 7 & $B$ & 4 & 19 & No & No \\
\hline 3 & 31 & C 8 & $\mathrm{~B}$ & 12 & 18 & No & No \\
\hline 4 & 36 & Th 3 & $A$ & 4 & 21 & No & Cholecystectomy \\
\hline 5 & 45 & C 7 & $A$ & 2 & 19 & No & Colostomy ${ }^{a}$ \\
\hline 6 & 47 & Th 8 & $A$ & 2 & 19 & No & Malones procedure ${ }^{b}$ \\
\hline 7 & 52 & Th 4 & $A$ & 16 & 21 & Hemorrhoids & No \\
\hline 8 & 55 & Th 9 & $A$ & 1 & 19 & No & No \\
\hline 9 & 62 & Th 9 & $A$ & 18 & 19 & No & No \\
\hline 10 & 64 & C 5 & B & 5 & 19 & No & No \\
\hline
\end{tabular}

Abbreviations: AIS, American Spinal Injury Association Impairment Scale; SCI, spinal cord injury.

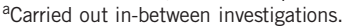

bCarried out in-between investigations. Use of procedure was discontinued shortly following surgery, hence not used at both investigations.

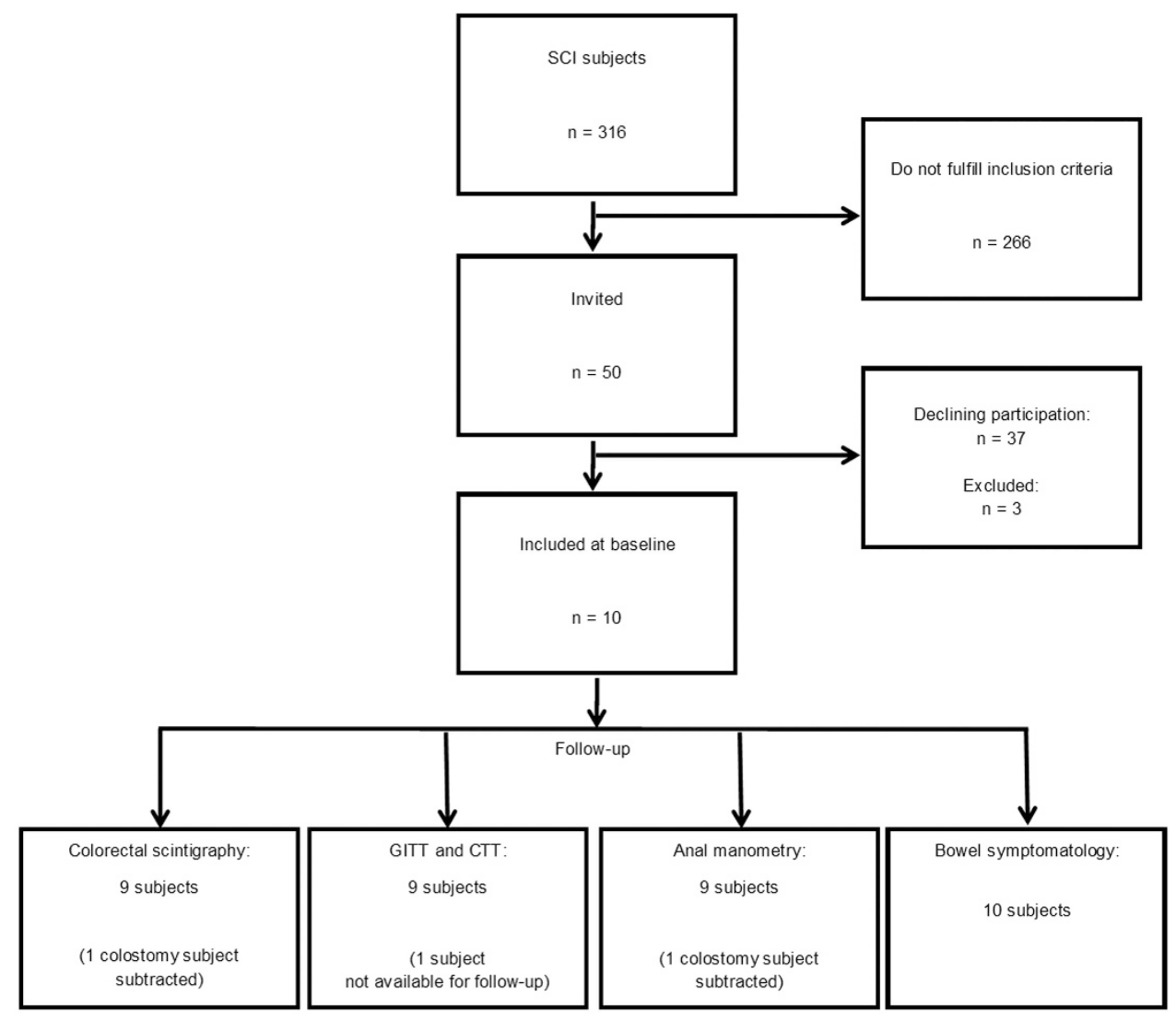

Figure 2 Flowchart of subject inclusion.

admission time was 3.5 days (range 3-14 days). One subject who had cerebrospinal fluid leakage underwent a dural closure procedure and was discharged 6 days later. Subsequently, he had reduced genital sensation ipsilateral to nerve route anastomosis and his erectile dysfunction worsened. One subject suffered a cerebrovascular accident within $24 \mathrm{~h}$ after surgery but fully recovered without any detectable sequelae. All other subjects had uneventful in-hospital stays with no surgical complications.

Events between baseline and follow-up

Owing to intolerable bowel symptoms, two subjects had to have surgery before follow-up. One subject had a colostomy 17 months after surgery and, consequently, most follow-up investigations were not performed. One had an appendicostomy for antegrade colonic enema 7 months after creation of the anastomosis, but the appendicostomy did not improve his bowel function and use was discontinued a few months after surgery and thus this subject was included in analysis

\section{Gastrointestinal and segmental colonic transit times}

One subject was not available for follow-up and, hence, analysis was made on the remaining nine subjects. No significant differences were observed between baseline and follow-up (Table 2). 


\section{Colorectal transport}

Ten subjects underwent baseline investigations of whom nine underwent follow-up (Figure 2). No scintigrams had segments overlapping but in some individuals, single segments were excluded for analysis because they had less than the required segmental counts prior to defecation (Table 3). Neither colorectal emptying during defecation or segmental colorectal transport at defecation showed any significant change from baseline to evaluation at 18 months (Figure 3 and Table 3). Correspondingly, stimulation of the dermatome intended for the reflex did not cause any movement of colorectal contents.

\section{Anorectal physiology}

Nine subjects were available for follow-up (Figure 2). No significant changes were seen in mean anal resting pressure (baseline: $61 \mathrm{~cm} \mathrm{H} \mathrm{H}_{2} \mathrm{O}$ (41-72 $\left.\mathrm{cm} \mathrm{H}_{2} \mathrm{O}\right)$ vs follow-up: $\left.72\left(57-84 \mathrm{~cm} \mathrm{H}_{2} \mathrm{O}\right), P=0.51\right)$ or mean anal pressure during attempted squeeze (baseline: $67 \mathrm{~cm} \mathrm{H}_{2} \mathrm{O}$ (36-73 cm $\left.\mathrm{H}_{2} \mathrm{O}\right)$ vs follow-up: $68 \mathrm{~cm} \mathrm{H}_{2} \mathrm{O} \quad\left(49-73 \mathrm{~cm} \mathrm{H}_{2} \mathrm{O}\right)$, $P=0.64)$. A borderline increase in rectal capacity was observed (baseline: $285 \mathrm{ml}(212-322 \mathrm{ml})$ vs follow-up: $336 \mathrm{ml}$ (95\% confidence interval: $312-489), P=0.06)$. Three subjects had sensory incomplete spinal cord lesions and thus some anorectal sensory function at baseline. They did not have any noticeable change at follow-up.

\section{Bowel symptoms}

All subjects were available for follow-up (Figure 2). However, the subject who had a colostomy filled-in the questionnaires 15 months after creation of the reflex arch just before having the stoma. Two of the 10 subjects reported that they felt bowel emptying had improved after creation of the artificial somatosensory reflex arch. However, no systematic differences were found in NBD score, St
Marks incontinence score or Cleveland constipation score (Table 4). The time spent for bowel care $(P=1.0)$ and the frequency of defecation $(P=0.75)$ did not change significantly either.

\section{DISCUSSION}

In contrast to our hypothesis and to previous reports, the creation of a somato-autonomic reflex arch had no effect on bowel function in subjects with supraconal SCI. For ethical reasons, only few subjects were included in this pilot study. The subjects, however, underwent comprehensive evaluation using previously validated objective methods and questionnaires before surgery and after 18 months follow-up.

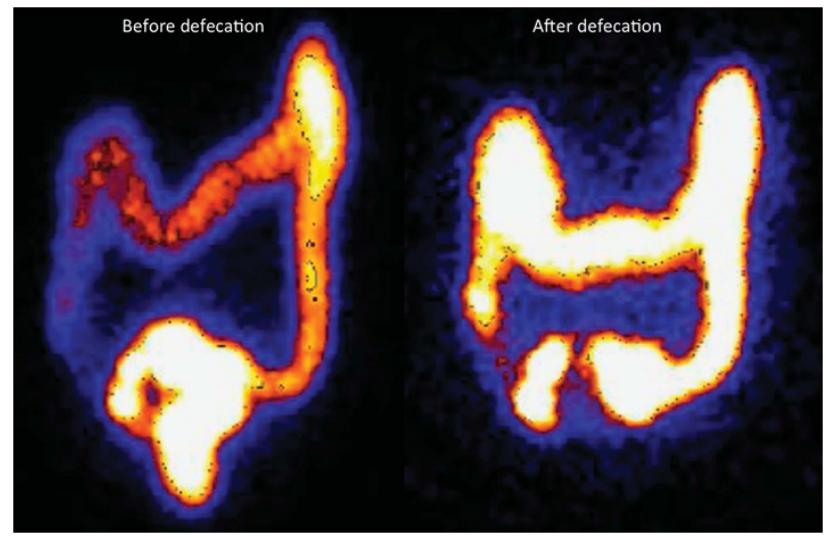

Figure 3 Colorectal scintigraphy illustration. Counts are color displayed. On the left, the situation prior to defecation; on the right, post defecation. Counts are lost in the recto-sigmoid corresponding to a median defecation score at baseline were 31 and at follow-up $75(n=5), P=0.50$.

Table 2 Segmental colonic and total gastrointestinal transit times at baseline and at follow-up

\begin{tabular}{|c|c|c|c|c|c|}
\hline Ascending colonic transit time (days) & 1.0 & $(0.6-2.1)$ & 0.5 & $(0.3-2.0)$ & 0.21 \\
\hline Transverse colonic transit time (days) & 0.6 & $(0.4-0.7)$ & 0.5 & $(0.1-0.5)$ & 0.17 \\
\hline Rectosigmoid transit time (days) & 0.6 & $(0.3-0.8)$ & 0.5 & $(0.1-0.8)$ & 0.86 \\
\hline Gastrointestinal transit time (days) & 4.8 & $(2.9-5.1)$ & 3.1 & $(2.7-4.8)$ & 0.77 \\
\hline
\end{tabular}

Wilcoxon signed rank test was applied.

Table 3 Emptying and colonic transport during defecation $(n=9)$. Wilcoxon signed rank test was applied

\begin{tabular}{|c|c|c|c|c|c|c|c|c|}
\hline & $n^{a}$ & Baseline (median) & Range (25-75 \%) & $n^{a}$ & Follow-up (median) & Range (25-75 \%) & $\mathrm{n}^{\mathrm{a}}$ & $P$ \\
\hline Defecation score & 7 & 31 & $(3.0-50)$ & 7 & 75 & $(30-153)$ & 5 & 0.50 \\
\hline \multicolumn{9}{|c|}{ Anterograd transport during defecation ${ }^{\mathrm{b}}$ : } \\
\hline Ascending & 10 & 4.5 & $(0.0-10)$ & 8 & 0.0 & $(0.0-8.5)$ & 8 & 0.36 \\
\hline Transverse & 10 & 6.0 & $(0.0-28)$ & 8 & 20.5 & $(6.0-38)$ & 8 & 0.89 \\
\hline Descending & 10 & 12 & $(1.0-22)$ & 9 & 17 & $(11-59)$ & 9 & 0.29 \\
\hline Recto-sigmoid & 7 & 31 & $(3.0-50)$ & 7 & 75 & $(30-229)$ & 5 & 0.50 \\
\hline \multicolumn{9}{|c|}{ Retrograd transport during defecation ${ }^{\mathrm{b}}$ : } \\
\hline Transverse & 10 & 0.0 & $(-3.0-0.0)$ & 8 & -4.0 & $(-4.5-(-0.5))$ & 8 & 0.26 \\
\hline Descending & 10 & 0.0 & $(0.0-0.0)$ & 9 & 0.0 & $(0.0-0.0)$ & 9 & 0.67 \\
\hline Recto-sigmoid & 7 & 0.0 & $(-1.0-0.0)$ & 7 & 0.0 & $(-3.0-0.0)$ & 5 & 0.79 \\
\hline
\end{tabular}

$a_{n}=$ number of subjects analyzed. All subjects with counts $<7.5 \%$ in analyzed segment was excluded for the individual calculation. For $P$-values, paired data were evaluated using Wilcoxon signed rank test.

bercentage of content moved of pre-defecatory counts. 
Table 4 Bowel function scores $(n=10)$

\begin{tabular}{lccrr}
\hline & Baseline (median) & Range (25-75 \%) & Follow-up (median) & Range (25-75 \%) \\
\hline Neurogenic bowel dysfunction score & 13.5 & $(12-16)$ & 12.5 & $(8.0-20)$ \\
St Marks incontinence score & 4.5 & $(2.0-6.0)$ & 5.0 & 0.51 \\
Cleveland constipation score & 6.0 & $(5.0-9.0)$ & 8.0 & 0.36 \\
\hline
\end{tabular}

Wilcoxon signed rank test was applied.

Approximately 2000 subjects have undergone the procedure internationally, especially in China. ${ }^{5}$ The main indication for the procedure is neurogenic bladder dysfunction, but it is commonly stated that it also alleviates NBD. ${ }^{2,5}$ Worldwide, a very large number of subjects suffer from neurogenic bladder and bowel dysfunction after SCI. Often treatment of bowel function is unsatisfactory and new treatment modalities are patently needed. Unfortunately, the surgically created somato-autonomic reflex arch was without effect and our recent report on the same subjects showed no clinically relevant effect on bladder function either. ${ }^{3}$ Consequently, we found that the procedure should be abandoned, at least for subjects with supraconal SCI.

The parasympathetic nervous system control of the gastrointestinal tract is mediated via the vagal nerve, excluding the descending colon, sigmoid, rectal canal, the anal canal and the internal anal sphincter which is innervated from the spinal cord via the sacral nerve roots (S2-S4). It modulates the enteric nervous system lying in the wall of the whole gastrointestinal tract. Parasympathetic stimulation increases peristaltic activity. Consequently, by way of stimulation of the L5(S1) dermatome the proposed mechanism of action for the somatoautonomic reflex arch for neurogenic constipation should be reflex activity in the sacral segment which in turn should lead to colorectal contractions in the left colon. Therefore, we used scintigraphy to assess colorectal transport both during mechanical stimulation and during subsequent defecation. Additionally, we evaluated the total effect on colorectal transit time with radiopaque markers. Both methods are well established and have been used to describe NBD and effects of treatment in previous studies. ${ }^{8}$ No effect was detected with either of the methods. Although the defecation score seemingly increased, it did not reach statistical significance. We cannot exclude a type II error, although the negative findings was supported by the remaining results. Either method has its limitations, the primary in this study being insufficient segmental counts prohibiting computation of movement of stool in some individuals.

As expected, the somato-autonomic reflex arch had no effect on anal pressure. Subjects with supraconal SCI have increased rectal tone ${ }^{17}$ and a trend towards larger rectal capacity was observed after the creation of the reflex arch. This can probably be explained by partial denervation of the rectum as one of the sacral roots was used for the anastomosis. It is unlikely that increased rectal capacity will reduce symptoms of constipation in subjects with SCI.

Two of the 10 subjects reported a sense of improved bowel emptying after the procedure. They considered the improvement very minor and it was not reflected in the bowel dysfunction scores. In general, no consistent improvement was reported through the NBD score, the St Marks fecal incontinence score or the Cleveland constipation score. All three scores are commonly used and the first is especially developed and validated for subjects with SCI. ${ }^{14}$

A potential risk of bias could have been that our surgical procedure had failed for technical reasons. Speaking against this, is the fact that we strived to perform the procedure exactly as described in the original publications and after observing it performed by its inventor in China. ${ }^{2}$ The procedure was performed by an experienced neurosurgeon with special expertise in spinal surgery and under close monitoring of electrophysiological responses during the procedure. As illustrated by our small series, surgery on subjects with SCI is not without risk and therefore it is crucial to always consider potential benefits and that they outweigh probable complications.

It is reported that reinnervation through the reflex arch occurs within 12 months. We chose to have follow-up after 18 months. Though not presented in the present study, some subjects have been followed for up to 42 months, still without effect. We considered the procedure experimental, and for ethical reasons, we chose only to include adult subjects with complete motor supraconal SCI as we would not risk subjects losing any remaining motor function due to transsection of the L5 motor root. Our choice of supraconal SCI was carried out to closely copy the procedure, as it has targeted these subjects. $^{2}$

The viability of the somato-autonomic reflex arch is supported by animal studies where anal sphincter contraction was observed during stimulation of the dermatome providing sensory innervation to the reflex. ${ }^{18}$ In 2012, 1890 subjects with spinal bifida and 306 with SCI had undergone the procedure in China. ${ }^{5}$ A few subjects have had the procedure outside China and data are available from a small group of American subjects. ${ }^{19,20}$ In Chinese cases available for evaluation, improved bowel function was reported by as many as $84.9 \%$. This is in stark contrast to our results. We cannot explain this extreme difference, but consider our results valid as they included comprehensive state-of-the-art evaluation of subjects before and after surgery.

In conclusion, we have performed comprehensive evaluation of bowel function in a small group of subjects with supraconal SCI treated with the surgically created somato-autonomic reflex arch. All subjects had NBD before the procedure and no clinically relevant changes in symptoms or colonic and anorectal function after surgery.

\section{DATA ARCHIVING}

There were no data to deposit.

\section{CONFLICT OF INTEREST}

The authors declare no conflict of interest.

\section{ACKNOWLEDGEMENTS}

The study was supported by the Lundbeck Foundation.

1 Krogh K, Nielsen J, Djurhuus JC, Mosdal C, Sabroe S, Laurberg S. Colorectal function in patients with spinal cord lesions. Dis Colon Rectum 1997; 40: 1233-1239.

2 Xiao C, Du M, Dai C, Li B, Nitti VW, de Groat WC. An artificial somatic-central nervous system-autonomic reflex pathway for controllable micturition after spinal cord injury: preliminary results in 15 patients. J Urol 2003; 170: 1237-1241.

3 Rasmussen MM, Rawashdeh YF, Clemmensen D, Tankisi H, Fuglsang-Frederiksen A, Krogh $\mathrm{K}$ et al. The artificial somato-autonomic reflex arch does not improve lower urinary tract function in patients with spinal cord lesions. J Urol 2014; 193: 598-604. 
4 Xiao C, Du M, Li B, Liu Z, Chen M, Chen Z et al. An artificial somatic-autonomic reflex pathway procedure for bladder control in children with spina bifida. J Urol 2005; 173 2112-2116.

5 Xiao CG. Xiao procedure for neurogenic bladder in spinal cord injury and spina bifida. Curr Bladder Dysfunct Rep 2012 2012/ 7: 83-87.

6 Abrahamsson H, Antov S, Bosaeus I. Gastrointestinal and colonic segmental transit time evaluated by a single abdominal $x$-ray in healthy subjects and constipated patients. Scand J Gastroenterol Supp/ 1988; 152: 72-80.

7 Krogh K, Olsen N, Christensen P, Madsen JL, Laurberg S. Colorectal transport during defecation in patients with lesions of the sacral spinal cord. Neurogastroenterol Motil 2003; 15: 25-31.

8 Rasmussen MM, Krogh K, Clemmensen D, Bluhme H, Rawashdeh Y, Christensen P. Colorectal transport during defecation in subjects with supraconal spinal cord injury. Spinal Cord 2013; 51: 683-687.

9 Hardy JG, Perkins AC. Validity of the geometric mean correction in the quantification of whole bowel transit. Nucl Med Commun 1985; 6: 217-224.

10 Krogh K, Olsen N, Christensen P, Madsen JL, Laurberg S. Colorectal transport in normal defaecation. Colorectal Dis 2003; 5: 185-192.

11 Ryhammer AM, Laurberg S, Hermann AP. Test-retest repeatability of anorectal physiology tests in healthy volunteers. Dis Colon Rectum 1997; 40: 287-292.
12 Krogh K, Perkash I, Stiens SA, Biering-Sorensen F. International bowel function basic spinal cord injury data set. Spinal Cord 2009; 47: 230-234.

13 Krogh K, Perkash I, Stiens SA, Biering-Sorensen F. International bowel function extended spinal cord injury data set. Spinal Cord 2009; 47: 235-241.

14 Krogh K, Christensen P, Sabroe S, Laurberg S. Neurogenic bowel dysfunction score. Spinal Cord 2006; 44: 625-631.

15 Vaizey CJ, Carapeti E, Cahill JA, Kamm MA. Prospective comparison of faecal incontinence grading systems. Gut 1999; 44: 77-80.

16 Agachan F, Chen T, Pfeifer J, Reissman P, Wexner SD. A constipation scoring system to simplify evaluation and management of constipated patients. Dis Colon Rectum 1996; 39: 681-685.

17 Krogh K, Mosdal C, Gregersen H, Laurberg S. Rectal wall properties in patients with acute and chronic spinal cord lesions. Dis Colon Rectum 2002; 45: 641-649.

18 Sun F, Chen M, Li W, Xiao C. Effect of the artificial somato-autonomic neuroanastomosis on defecation after spinal cord injury and its underlying mechanisms. J Huazhong Univ Sci Technolog Med Sci 2010; 30: 490-493.

19 Peters KM, Girdler B, Turzewski C, Trock G, Feber K, Nantau W et al. Outcomes of lumbar to sacral nerve rerouting for spina bifida. J Urol 2010; 184: 702-707.

20 Tuite GF, Storrs BB, Homsy YL, Gaskill SJ, Polsky EA, Reilly MA et al. Attempted bladder reinnervation and creation of a scratch reflex for bladder emptying through a somatic-to-autonomic intradural anastomosis. J Neursurg Pediatr 2013; 12: 80-86. 